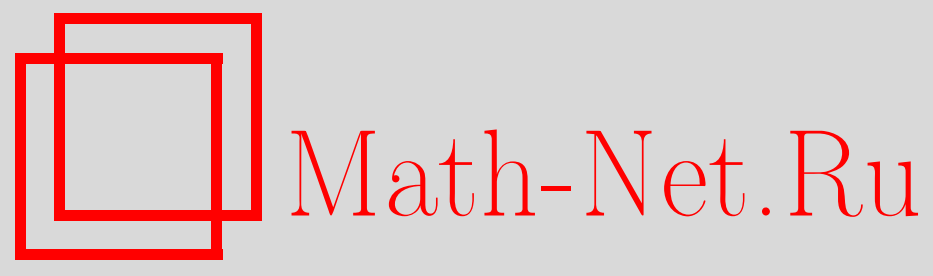

А. С. Холево, М. Е. Широков, Непрерывные ансамбли и пропускная способность квантовых каналов бесконечной размерности, Теория вероятн. и ее примен., 2005, том 50, выпуск 1, 98-114

DOI: https://doi.org/10.4213/tvp160

Использование Общероссийского математического портала MathNet.Ru подразумевает, что вы прочитали и согласны с пользовательским соглашением

http: //www . mathnet.ru/rus/agreement

Параметры загрузки:

IP : 3.85 .7 .115

26 апреля 2023 г., $12: 22: 26$

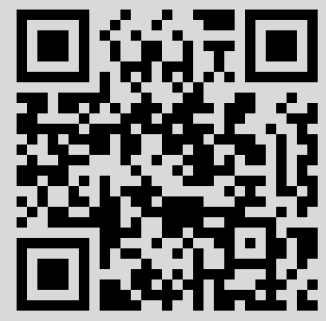




\title{
НЕПРЕРЫВНЫЕ АНСАМБЛИ И ПРОПУСКНАЯ СПОСОБНОСТЬ КВАНТОВЫХ КАНАЛОВ БЕСКОНЕЧНОЙ РАЗМЕРНОСТИ ${ }^{1)}$
}

\begin{abstract}
Работа посвящена изучению $\chi$-пропускной способности, тесно связанной с пропускной способностью для передачи классической информации по квантовому каналу связи бесконечной размерности. Для таких каналов обобщенные входные ансамбли определяются как вероятностные меры на множестве всех квантовых состояний. Доказана компактность множества всех обобщенных ансамблей, средние которых принадлежат произвольному компактному подмножеству состояний. Этот результат позволяет получить достаточное условие существования оптимального обобщенного ансамбля для бесконечномерных квантовых каналов с ограничениями. Показано, что данное условие выполняется для бозонных гауссовских каналов с ограничениями на среднюю энергию. В случае ограничений, задаваемых выпуклыми множествами, получена характеризация оптимального обобшенного ансамбля, обобщающая свойство «максимальной удаленности».
\end{abstract}

Ключевые слова и фразы: квантовый канал, $\chi$-пропускная способность, обобщенные ансамбли.

1. Введение. Настоящая работа посвящена систематическому изучению $\chi$-пропускной способности ${ }^{2)}$ квантовых каналов бесконечной размерности и является логическим продолжением работ [8], [10], [17]. Хотя в настоящее время основное место в исследованиях по квантовой теории информации занимают конечномерные квантовые системы, имеется важный и интересный класс гауссовских каналов (см., например, [9], [4], [16]), которые действуют в бесконечномерных гильбертовых пространствах. Известно, что ряд вопросов для гауссовских систем с конечным

* Математический институт им. В. А. Стеклова РАН, ул. Губкина, 8, 119991 Москва, ГСП-1, Россия; e-mail: holevo@mi.ras.ru; msh@mi.ras.ru

1) Работа выполнена при частичной поддержке грантов НШ 1758.2003.1, ИНТАС 00-738. Авторы выражают благодарность организаторам программы «Квантовая теория информации» в Институте Исаака Ньютона (Кембридж), в рамках которой данная работа получила завершение.

2) Эта величина, называемая в зарубежной литературе the Holevo capacity, тесно связана с пропускной способностью для передачи классической информации по квантовому каналу связи, см., например, [6]. 
числом степеней свободы решается методами конечномерной линейной алгебры, однако при обшем и математически строгом подходе нельзя избежать бесконечномерного анализа этих систем. Далее, как было отмечено в [17], знаменитое доказательство эквивалентности различных форм гипотезы аддитивности в квантовой теории информации, данное П. Шором [18], тесно связано со свойством разрывности $\chi$-пропускной способности как функции канала в бесконечномерном случае. Исследование всех этих вопросов требует привлечения результатов теории операторов в гильбертовом пространстве и теории меры в метрических пространствах.

Для каналов бесконечной размерности характерны следующие две особенности. Первая состоит в необходимости введения ограничений на входные ансамбли состояний (таких как ограничение средней энергии для гауссовских каналов), хотя, как показано в [10], входные ограничения оказываются полезными и при исследовании гипотезы аддитивности для каналов конечной размерности. Другая особенность состоит в естественном появлении бесконечных, в общем случае «непрерывных» ансамблей, которые определяются как вероятностные меры на множестве всех квантовых состояний. Используя известный в теории вероятностей критерий компактности и другие факты теории меры, можно доказать компактность множества всех обобщенных ансамблей, средние которых принадлежат произвольному компактному подмножеству состояний. Этот результат позволяет получить достаточное условие существования оптимального обобщенного ансамбля для бесконечномерных квантовых каналов с ограничениями.

Показано, что данное условие выполняется для бозонных гауссовских каналов с ограничениями на среднюю энергию. В случае ограничений, задаваемых выпуклыми множествами, получена характеризация оптимального обобщенного ансамбля, обобщающая свойство «максимальной удаленности» (см. [15], [10]).

2. Предварительные сведения. Ниже кратко приводятся некоторые сведения из некоммутативной теории вероятностей, подробнее см. в [7], [14]. Пусть $\mathscr{H}$ - сепарабельное гильбертово пространство, $\mathfrak{B}(\mathscr{H})$ - алгебра всех ограниченных операторов в $\mathscr{H}, \mathfrak{T}(\mathscr{H})$ - банахово пространство всех ядерных операторов со следовой нормой $\|\cdot\|_{1}$. Состоянием называется положительный ядерный оператор $\rho$ в $\mathscr{H}$ с единичным следом: $\rho \geqslant 0, \operatorname{Tr} \rho=1$. Алгебра $\mathfrak{B}(\mathscr{H})$ обычно называется алгеброй наблюдаемых квантовой системы, тогда состояние задает функционал математического ожидания $A \mapsto \operatorname{Tr} \rho A, A \in \mathfrak{B}(\mathscr{H})$. Множество всех состояний $\mathfrak{S}(\mathscr{H})$ - выпуклое замкнутое подмножество $\mathfrak{T}(\mathscr{H})$, которое является полным сепарабельным метрическим пространством с метрикой, определяемой следовой нормой. 
Далее неоднократно будет использован следующий результат, полученный в [1]: сходимость последовательности состояний к некоторому состоянию в слабой операторной топологии эквивалентна сходимости этой последовательности по следовой норме. Отметим также следующую характеристику компактных подмножеств состояний [14] (некоммутативный аналог теоремы Прохорова): замкнутое подмножество $\mathscr{K}$ множества $\mathfrak{S}(\mathscr{H})$ компактно тогда и только тогда, когда для любого $\varepsilon>0$ найдется проектор $P$ конечного ранга такой, что $\operatorname{Tr} \rho P \geqslant 1-\varepsilon$ для любого $\rho \in \mathscr{K}$. Доказательство этого утверждения приведено в приложении А.

Конечный набор $\left\{\pi_{i}, \rho_{i}\right\}$ состояний $\rho_{i}$ с соответствующими вероятностями $\pi_{i}$ называется ансамблем, а состояние $\bar{\rho}=\sum_{i} \pi_{i} \rho_{i}-$ средним этого ансамбля.

В вопросах, касающихся теории вероятностных мер на сепарабельных метрических пространствах, мы будем следовать [2], [13]. В частности, в определении носителя $\operatorname{supp}(\pi)$ меры $\pi$ мы следуем [13].

О п р е д е л е н и е. Обобщенным ансамблем называется произвольная вероятностная борелевская мера $\pi$ на $\mathfrak{S}(\mathscr{H})$. Средним обобшенного ансамбля $\pi$ называется состояние ${ }^{3)}$, определяемое интегралом Петтиса

$$
\bar{\rho}(\pi)=\int_{\mathfrak{S}(\mathscr{H})} \rho \pi(d \rho) .
$$

Используя упомянутый выше результат из [1], нетрудно показать, что данный интеграл существует и как интеграл Бохнера (см. [5]), т.е. в смысле ядерной нормы. Обычное понятие ансамбля соответствует мерам с конечным носителем.

Обозначим $\mathscr{P}$ выпуклое множество всех вероятностных мер на $\mathfrak{S}(\mathscr{H})$, снабженное топологией слабой сходимости (см. [2]). Отображение $\pi \mapsto \bar{\rho}(\pi)$ непрерывно в этой топологии. В самом деле, слабая сходимость последовательности $\left\{\pi_{n}\right\} \subset \mathscr{P}$ к $\pi \in \mathscr{P}$ влечет сходимость последовательности состояний $\left\{\bar{\rho}\left(\pi_{n}\right)\right\}$ к состоянию $\bar{\rho}(\pi)$ в слабой операторной топологии, откуда в силу результата из [1] следует сходимость в смысле следовой нормы.

Лемма 1. Множество мер с конечньм носителем является плотным подмножеством множества всех мер с заданным средним $\bar{\rho}$.

Доказательство данной леммы приведено в приложении Б.

Будем использовать символ $\log$ для обозначения функции на $[0,+\infty)$, совпадающей с обычным логарифмом на $(0,+\infty)$ и равной нулю в нуле. Для положительного оператора $A$ конечного ранга определим энтропию формулой

$$
H(A)=\operatorname{Tr} A(I \log \operatorname{Tr} A-\log A),
$$

3) Это состояние называется также барицентром меры $\pi$. 
где $I$ - тождественный оператор в $\mathscr{H}$. В частности, энтропия состояния $\rho$ (энтропия фон Неймана) равна

$$
H(\rho)=-\operatorname{Tr} \rho \log \rho .
$$

Относительную энтропию двух положительных операторов $A$ и $B$ конечного ранга определим выражением

$$
H(A \| B)=\operatorname{Tr}(A \log A-A \log B+B-A)
$$

при условии $\operatorname{ran} A \subseteq \operatorname{ran} B$ и положим $H(A \| B)=+\infty$ в противном случае (символ ran обозначает замыкание множества значений оператора).

Данные определения можно расширить на произвольные положительные ядерные операторы $A$ и $B$ с помощью следующей леммы (cM. [11]).

Лемма 2. Пусть $\left\{P_{n}\right\}$ - произвольная последовательность проекторов конечного ранга, монотонно возрастающая $\kappa$ в в сильной операторной топологии. Последовательности $\left\{H\left(P_{n} A P_{n}\right)\right\}$, $\left\{H\left(P_{n} A P_{n} \| P_{n} B P_{n}\right)\right\}$ являются неубьваюшими, и их предельи (конечнье или бесконечные) не зависят от выбора последовательности $\left\{P_{n}\right\}$.

Используя данную лемму, можно определить энтропию и относительную энтропию следующим образом:

$$
H(A)=\lim _{n \rightarrow+\infty} H\left(P_{n} A P_{n}\right), \quad H(A \| B)=\lim _{n \rightarrow+\infty} H\left(P_{n} A P_{n} \| P_{n} B P_{n}\right) .
$$

Как хорошо известно, свойства энтропии в конечномерном и бесконечномерном случаях существенно различны: в первом случае энтропия является непрерывной ограниченной функцией на $\mathfrak{S}(\mathscr{H})$, а во втором она разрывна (полунепрерывна снизу) в каждой точке и бесконечна «почти всюду» в том смысле, что множество состояний с конечной энтропией является подмножеством первой категории в $\mathfrak{S}(\mathscr{H})$ (см. [19]).

3. $\chi$-пропускная способность канала с ограничением. Пусть $\mathscr{H}, \mathscr{H}^{\prime}$ - пара сепарабельных гильбертовых пространств, которые мы будем называть входным и выходным пространством соответственно. Канал $\Phi$ - это линейное положительное сохраняющее след отображение $\mathfrak{T}(\mathscr{H})$ в $\mathfrak{T}\left(\mathscr{H}^{\prime}\right)$ такое, что двойственное отображение $\Phi^{*}: \mathfrak{B}\left(\mathscr{H}^{\prime}\right) \mapsto \mathfrak{B}(\mathscr{H})$ (которое существует, поскольку $\Phi$ ограничено) является вполне положительным, см. [7]. В частности, канал отображает (входные) состояния в $\mathscr{H}$ в выходные состояния в $\mathscr{H}^{\prime}$.

Пусть $\mathscr{A}$ - произвольное подмножество $\mathfrak{S}(\mathscr{H})$. Рассмотрим ограничение на входной ансамбль $\left\{\pi_{i}, \rho_{i}\right\}$, определяемое включением $\bar{\rho} \in \mathscr{A}$. Канал $\Phi$ с таким ограничением называется $\mathscr{A}$-ограниченнылм каналом. Определим $\chi$-пропускную способность $\mathscr{A}$-ограниченного канала $\Phi$ следующим образом:

$$
\bar{C}(\Phi ; \mathscr{A})=\sup _{\bar{\rho} \in \mathscr{A}} \chi_{\Phi}\left(\left\{\pi_{i}, \rho_{i}\right\}\right)
$$


где

$$
\chi_{\Phi}\left(\left\{\pi_{i}, \rho_{i}\right\}\right)=\sum_{i} \pi_{i} H\left(\Phi\left(\rho_{i}\right) \| \Phi(\bar{\rho})\right) .
$$

В данной статье рассматриваются такие множества-ограничения $\mathscr{A}$, что

$$
\bar{C}(\Phi ; \mathscr{A})<+\infty
$$

Подмножество $\mathscr{P}$, состоящее из всех мер $\pi$ со средним состоянием $\bar{\rho}(\pi)$ в $\mathscr{A} \subseteq \mathfrak{S}(\mathscr{H})$, обозначим $\mathscr{P} \mathscr{A}$.

В силу леммы 2 неотрицательная функция $\rho \mapsto H(\Phi(\rho) \| \Phi(\bar{\rho}(\pi)))$ измерима на $\mathfrak{S}(\mathscr{H})$. Следовательно, функционал

$$
\chi_{\Phi}(\pi)=\int_{\mathfrak{S}(\mathscr{H})} H(\Phi(\rho) \| \Phi(\bar{\rho}(\pi))) \pi(d \rho)
$$

определен на множестве $\mathscr{P}($ с множеством значений $[0 ;+\infty])$.

Предложение 1. Функиионал $\chi_{\Phi}(\pi)$ полунепрерывен снизу на множестве $\mathscr{P}$. Если $H\left(\Phi\left(\bar{\rho}\left(\pi^{\prime}\right)\right)\right)<\infty$, то

$$
\chi_{\Phi}(\pi)=H(\Phi(\bar{\rho}(\pi)))-\int_{\mathfrak{S}(\mathscr{H})} H(\Phi(\rho)) \pi(d \rho) .
$$

Д ок аз а т ель с т в о. Пусть $\left\{P_{n}\right\}$ - произвольная последовательность проекторов конечного ранга, монотонно возрастающая к $I$. Покажем, что функционалы

$$
\chi_{\Phi}^{n}(\pi)=\int_{\mathfrak{S}(\mathscr{H})} H\left(P_{n} \Phi(\rho) P_{n} \| P_{n} \Phi(\bar{\rho}(\pi)) P_{n}\right) \pi(d \rho)
$$

непрерывны на множестве $\mathscr{P}$.

Заметим, что

$$
\operatorname{ran}\left(P_{n} \Phi(\rho) P_{n}\right) \subseteq \operatorname{ran}\left(P_{n} \Phi(\bar{\rho}(\pi)) P_{n}\right)
$$

для $\pi$-почти всех $\rho$. Действительно, замыкание множества значений является ортогональным дополнением ядра эрмитова оператора, а для ядер противоположное включение очевидно. Следовательно,

$$
\begin{aligned}
& H\left(P_{n} \Phi(\rho) P_{n} \| P_{n} \Phi(\bar{\rho}(\pi)) P_{n}\right) \\
& \quad=\operatorname{Tr}\left(P_{n} \Phi(\rho) P_{n} \log \left(P_{n} \Phi(\rho) P_{n}\right)-P_{n} \Phi(\rho) P_{n} \log \left(P_{n} \Phi(\bar{\rho}(\pi)) P_{n}\right)\right. \\
& \left.\quad+P_{n} \Phi(\bar{\rho}(\pi)) P_{n}-P_{n} \Phi(\rho) P_{n}\right)
\end{aligned}
$$

для $\pi$-почти всех $\rho$. Используя $(1)$, получаем

$$
\begin{aligned}
\chi_{\Phi}^{n}(\pi)= & -\int_{\mathfrak{S}(\mathscr{H})} H\left(P_{n} \Phi(\rho) P_{n}\right) \pi(d \rho) \\
& +\int_{\mathfrak{S}(\mathscr{H})} \operatorname{Tr}\left(P_{n} \Phi(\rho)\right) \log \operatorname{Tr}\left(P_{n} \Phi(\rho)\right) \pi(d \rho)
\end{aligned}
$$




$$
\begin{aligned}
& -\int_{\mathfrak{S}(\mathscr{H})} \operatorname{Tr}\left(P_{n} \Phi(\rho) P_{n}\right) \log \left(P_{n} \Phi(\bar{\rho}(\pi)) P_{n}\right) \pi(d \rho) \\
& +\int_{\mathfrak{S}(\mathscr{H})} \operatorname{Tr}\left(P_{n} \Phi(\bar{\rho}(\pi))\right) \pi(d \rho)-\int_{\mathfrak{S}(\mathscr{H})} \operatorname{Tr}\left(P_{n} \Phi(\rho)\right) \pi(d \rho) .
\end{aligned}
$$

Легко видеть, что два последних слагаемых сокращаются, а третье слагаемое можно преобразовать следуюшим образом:

$$
\begin{aligned}
& -\int_{\mathfrak{S}(\mathscr{H})} \operatorname{Tr}\left(P_{n} \Phi(\rho) P_{n}\right) \log \left(P_{n} \Phi(\bar{\rho}(\pi)) P_{n}\right) \pi(d \rho) \\
& \quad=-\operatorname{Tr} \int_{\mathfrak{S}(\mathscr{H})}\left(P_{n} \Phi(\rho) P_{n}\right) \log \left(P_{n} \Phi(\bar{\rho}(\pi)) P_{n}\right) \pi(d \rho) \\
& \quad=H\left(P_{n} \Phi(\bar{\rho}(\pi)) P_{n}\right)-\operatorname{Tr}\left(P_{n} \Phi(\bar{\rho}(\pi))\right) \log \operatorname{Tr}\left(P_{n} \Phi(\bar{\rho}(\pi))\right) .
\end{aligned}
$$

Следовательно,

$$
\begin{aligned}
\chi_{\Phi}^{n}(\pi)= & H\left(P_{n} \Phi(\bar{\rho}(\pi)) P_{n}\right)-\operatorname{Tr}\left(P_{n} \Phi(\bar{\rho}(\pi))\right) \log \operatorname{Tr}\left(P_{n} \Phi(\bar{\rho}(\pi))\right) \\
& -\int_{\mathfrak{S}(\mathscr{H})} H\left(P_{n} \Phi(\rho) P_{n}\right) \pi(d \rho) \\
& +\int_{\mathfrak{S}(\mathscr{H})} \operatorname{Tr}\left(P_{n} \Phi(\rho)\right) \log \operatorname{Tr}\left(P_{n} \Phi(\rho)\right) \pi(d \rho) .
\end{aligned}
$$

Из непрерывности и ограниченности энтропии в конечномерном случае и аналогичных свойств функции $\rho \mapsto \operatorname{Tr}\left(P_{n} \Phi(\rho)\right) \log \operatorname{Tr}\left(P_{n} \Phi(\rho)\right)$ следует непрерывность функционалов $\chi_{\Phi}^{n}(\pi)$ при всех $n$.

В силу теоремы о монотонной сходимости последовательность функционалов $\chi_{\Phi}^{n}(\pi)$ является неубываюшей и поточечно сходится к $\chi_{\Phi}(\pi)$. Следовательно, функционал $\chi_{\Phi}(\pi)$ полунепрерывен снизу как верхняя грань семейства непрерывных функций.

Для доказательства (6) заметим, что из леммы 2 следует

$$
\lim _{n \rightarrow+\infty} H\left(P_{n} \Phi(\bar{\rho}(\pi)) P_{n}\right)=H(\Phi(\bar{\rho}(\pi)))
$$

и

$$
\lim _{n \rightarrow+\infty} \int_{\mathfrak{S}(\mathscr{H})} H\left(P_{n} \Phi(\rho) P_{n}\right) \pi(d \rho)=\int_{\mathfrak{S}(\mathscr{H})} H(\Phi(\rho)) \pi(d \rho)
$$

в силу теоремы о монотонной сходимости. Для любого $\rho$ последовательность $\left\{\operatorname{Tr}\left(P_{n} \Phi(\rho)\right)\right\}$ принадлежит $[0,1]$ и сходится к 1 , следовательно, $\lim _{n \rightarrow+\infty} \operatorname{Tr}\left(P_{n}(\rho)\right) \log \operatorname{Tr}\left(P_{n}(\rho)\right)=0$, в частности, второе слагаемое в $(7)$ стремится к 0 . Поскольку $|x \log x|<1$ для всех $x \in(0,1]$, последнее слагаемое в (7) также стремится к 0 по теореме о мажорируемой сходимости. Поэтому переход к пределу $n \rightarrow \infty$ в (7) дает (6). Предложение 1 доказано.

Следствие 1. $\chi$-пропускную способность $\mathscr{A}$-ограниченного канала $\Phi$ можно определить выражением

$$
\bar{C}(\Phi ; \mathscr{A})=\sup _{\pi \in \mathscr{P}_{\mathscr{A}}} \chi_{\Phi}(\pi) .
$$


Д о к а з а т е л ь с т в о. Определение (3) аналогично данному выражению, в котором верхняя грань берется по всем мерам из $\mathscr{P} \mathscr{A}$ с конечным носителем. В силу леммы 1 произвольную меру $\pi$ из $\mathscr{P} \mathscr{A}$ можно приблизить последовательностью мер $\left\{\pi_{n}\right\}$ из $\mathscr{P}_{\mathscr{A}}$, имеющих конечный носитель. В силу предложения $1 \liminf _{n \rightarrow+\infty} \chi_{\Phi}\left(\pi_{n}\right) \geqslant \chi_{\Phi}(\pi)$. Следовательно, верхняя грань по всем мерам из $\mathscr{P} \mathscr{A}$ совпадает с верхней гранью по всем мерам из $\mathscr{P}_{\mathscr{A}}$ с конечным носителем. Следствие 1 доказано.

4. Компактные ограничения. Для дальнейшего удобно ввести следующее определение: неограниченный положительный оператор $H$ в гильбертовом пространстве $\mathscr{H}$, имеющий дискретный спектр конечной кратности, будем называть $\mathfrak{H}$-оператором. Пусть $Q_{n}-$ спектральный проектор оператора $H$, соответствующий $n$ наименьшим собственным значениям. Следуя [8], обозначим

$$
\operatorname{Tr} \rho H=\lim _{n \rightarrow \infty} \operatorname{Tr} \rho Q_{n} H,
$$

где в правой части - неубывающая последовательность. В [8] доказано, что для произвольного $\mathfrak{H}$-оператора $H$ множество

$$
\mathscr{K}=\{\rho: \operatorname{Tr} \rho H \leqslant h\}
$$

является компактным подмножеством $\mathfrak{S}(\mathscr{H})$.

Лемма 3. Пусть $\mathscr{A}-$ компактное подмножество $\mathfrak{S}(\mathscr{H})$. Существует $\mathfrak{H}$-оператор $H$ и положительное число $h$ такие, что $\operatorname{Tr} \rho H \leqslant h$ $\partial \Omega$ в всех $\rho \in \mathscr{A}$.

Д о к а з а тел в с т в о. В силу общего критерия компактности (см. Приложение А) для любого натурального числа $n$ существует проектор $P_{n}$ конечного ранга такой, что $\operatorname{Tr} \rho P_{n} \geqslant 1-n^{-3}$ для всех $\rho$ из $\mathscr{A}$. Без ограничения общности можно считать, что $\bigvee_{k=1}^{+\infty} P_{k}(\mathscr{H})=\mathscr{H}$, где $\bigvee$ обозначает замыкание линейной оболочки подпространств. Пусть $\widehat{P}_{n}-$ проектор на конечномерное подпространство $\bigvee_{k=1}^{n} P_{k}(\mathscr{H})$. Таким образом, $H=\sum_{n=1}^{+\infty} n\left(\widehat{P}_{n+1}-\widehat{P}_{n}\right)-\mathfrak{H}$-оператор, удовлетворяющий условию

$$
\operatorname{Tr} \rho H=\sum_{n=1}^{+\infty} n \operatorname{Tr} \rho\left(\widehat{P}_{n+1}-\widehat{P}_{n}\right) \leqslant \sum_{n=1}^{+\infty} n \operatorname{Tr} \rho\left(I_{\mathscr{H}}-\widehat{P}_{n}\right) \leqslant \sum_{n=1}^{+\infty} n^{-2}=h
$$

для произвольного состояния $\rho$ из $\mathscr{A}$. Лемма 3 доказана.

Данную лемму можно использовать для доказательства компактности некоторых подмножеств состояний. Рассмотрим, например, множество $\mathscr{C}(\rho, \sigma)$ всех состояний $\omega$ в тензорном произведении гильбертовых пространств $\mathscr{H}$ и $\mathscr{K}$, имеюших заданные частичные следы $\operatorname{Tr}_{\mathscr{K}} \omega=\rho$ и $\operatorname{Tr}_{\mathscr{H}} \omega=\sigma$. В силу леммы 3 существуют такие $\mathfrak{H}$-операторы $A$ и $B$ в пространствах $\mathscr{H}$ и $\mathscr{K}$ соответственно, что $\operatorname{Tr} \rho A=\alpha<+\infty$ и $\operatorname{Tr} \sigma B=\beta<+\infty$. Нетрудно видеть, что $C=A \otimes I_{\mathscr{K}}+I_{\mathscr{H}} \otimes B-$ 
$\mathfrak{H}$-оператор в пространстве $\mathscr{H} \otimes \mathscr{K}$ и

$$
\operatorname{Tr} \omega C=\operatorname{Tr} \rho A+\operatorname{Tr} \sigma B=\alpha+\beta<+\infty \quad \forall \omega \in \mathscr{C}(\rho, \sigma) .
$$

Из замечания перед леммой 3 следует компактность множества $\mathscr{C}(\rho, \sigma)$.

Предложение 2. Множество $\mathscr{P} \mathscr{A}$ является компактным подмножеством ЯР тогда и только тогда, когда множество $\mathscr{A}$ является компактным подмножеством $\mathfrak{S}(\mathscr{H})$.

Д о к а з а т ел ь с т в о. Пусть множество $\mathscr{P}_{\mathscr{A}}$ компактно. Множество $\mathscr{A}$ является образом множества $\mathscr{P} \mathscr{A}$ при непрерывном отображении $\pi \mapsto \bar{\rho}(\pi)$ и, следовательно, компактно.

Пусть множество $\mathscr{A}$ компактно. В силу леммы 3 существует $\mathfrak{H}-$ оператор $H$ такой, что $\operatorname{Tr} \rho H \leqslant h$ для всех $\rho$ из $\mathscr{A}$. Для любой меры $\pi \in \mathscr{P}_{\mathscr{A}}$ имеем

$$
\int_{\mathfrak{S}(\mathscr{H})}(\operatorname{Tr} \rho H) \pi(d \rho)=\operatorname{Tr}\left(\int_{\mathfrak{S}(\mathscr{H})} \rho \pi(d \rho) H\right)=\operatorname{Tr} \bar{\rho}(\pi) H \leqslant h .
$$

Существование интеграла в левой части и первое равенство вытекают из теоремы о монотонной сходимости, поскольку в силу (8) функция $\operatorname{Tr} \rho H$ является пределом неубывающей последовательности непрерывных ограниченных функций $\operatorname{Tr} \rho Q_{n} H$.

Пусть $\mathscr{K}_{\varepsilon}=\left\{\rho: \operatorname{Tr} \rho H \leqslant h \varepsilon^{-1}\right\}$. Множество $\mathscr{K}_{\varepsilon}$ компактно при всех $\varepsilon$. В силу (10) для любой меры $\pi$ из $\mathscr{P} \mathscr{A}$ получаем

$$
\pi\left(\mathfrak{S}(\mathscr{H}) \backslash \mathscr{K}_{\varepsilon}\right)=\int_{\mathfrak{S}(\mathscr{H}) \backslash \mathscr{K}_{\varepsilon}} \pi(d \rho) \leqslant \varepsilon h^{-1} \int_{\mathfrak{S}(\mathscr{H}) \backslash \mathscr{K}_{\varepsilon}}(\operatorname{Tr} \rho H) \pi(d \rho) \leqslant \varepsilon .
$$

Компактность множества $\mathscr{P}_{\mathscr{A}}$ следует теперь из теоремы Прохорова (см. [12]). Предложение 2 доказано.

Нам потребуются следующие понятия, введенные в [17]. Последовательность ансамблей $\left\{\pi_{i}^{k}, \rho_{i}^{k}\right\}$ со средними состояниями $\bar{\rho}^{k} \in \mathscr{A}$ называется аппроксимирующей последовательностью, если

$$
\lim _{k \rightarrow+\infty} \chi_{\Phi}\left(\left\{\pi_{i}^{k}, \rho_{i}^{k}\right\}\right)=\bar{C}(\Phi ; \mathscr{A}) .
$$

Состояние $\bar{\rho} \in \mathscr{A}$ называется оптимальным средним для $\mathscr{A}$-ограниченного канала $\Phi$, если оно является частичным пределом последовательности средних некоторой аппроксимирующей последовательности ансамблей. Компактность множества $\mathscr{A}$ гарантирует существование оптимальных средних.

Теорема. Пусть $\mathscr{A}-$ компактное множество. Если сужение выходной энтропии $H(\Phi(\rho))$ на множество $\mathscr{A}$ непрерьвно хотя би в одном оптимальном среднем состоянии $\bar{\rho}_{0} \in \mathscr{A}$, то существует оптимальный обобщенный ансамбль $\pi^{*}$ из $\mathscr{P}_{\mathscr{A}}$ такой, что $\operatorname{supp} \pi^{*} \subseteq$ $\operatorname{Extr} \mathfrak{S}(\mathscr{H}) u$

$$
\bar{C}(\Phi ; \mathscr{A})=\chi_{\Phi}\left(\pi^{*}\right)=\int_{\mathfrak{S}(\mathscr{H})} H\left(\Phi(\rho) \| \Phi\left(\bar{\rho}\left(\pi^{*}\right)\right)\right) \pi^{*}(d \rho) .
$$


Д о к а з а т е л ь с т в о. Покажем, что функционал

$$
\pi \longmapsto \int_{\mathfrak{S}(\mathscr{H})} H(\Phi(\rho)) \pi(d \rho)
$$

определен и полунепрерывен снизу на множестве $\mathscr{P}$.

В силу леммы 2 функция $H(\Phi(\rho))$ есть поточечный предел неубывающей последовательности функций

$$
f_{n}(\rho)=\operatorname{Tr}\left(\left(P_{n} \Phi(\rho) P_{n}\right)\left(I \log \operatorname{Tr}\left(P_{n} \Phi(\rho) P_{n}\right)-\log \left(P_{n} \Phi(\rho) P_{n}\right)\right)\right),
$$

которые непрерывны и ограничены на $\mathfrak{S}(\mathscr{H})$. Следовательно, функция $H(\Phi(\rho))$ измерима, и в силу теоремы о монотонной сходимости

$$
\int_{\mathfrak{S}(\mathscr{H})} H(\Phi(\rho)) \pi(d \rho)=\lim _{n \rightarrow \infty} \int_{\mathfrak{S}(\mathscr{H})} f_{n}(\rho) \pi(d \rho) .
$$

Последовательность непрерывных функционалов

$$
\pi \longmapsto \int_{\mathfrak{S}(\mathscr{H})} f_{n}(\rho) \pi(d \rho)
$$

является неубывающей. Следовательно, ее поточечный предел полунепрерывен снизу.

По условию теоремы сужение функции $H(\Phi(\rho))$ на множество $\mathscr{A}$ непрерывно в некотором оптимальном среднем состоянии $\bar{\rho}_{0}$. Из непрерывности отображения $\pi \mapsto \bar{\rho}(\pi)$ следует, что сужение функционала $\pi \mapsto H(\Phi(\bar{\rho}(\pi)))$ на множество $\mathscr{P}_{\mathscr{A}}$ непрерывно в каждой точке $\pi_{0}$ такой, что $\bar{\rho}\left(\pi_{0}\right)=\bar{\rho}_{0}$. Поэтому $H(\Phi(\bar{\rho}(\pi)))<+\infty$ для любой точки $\pi$ из пересечения $\mathscr{P}_{\mathscr{A}}$ с некоторой окрестностью точки $\pi_{0}$. Для каждой такой точки $\pi$ имеет место (6). Следовательно, сужение функционала $\chi_{\Phi}(\pi)$ на множество $\mathscr{P}_{\mathscr{A}}$ полунепрерывно сверху, а значит, в силу предложения 1 , непрерывно в каждой точке $\pi_{0}$ из $\mathscr{P}_{\mathscr{A}}$ такой, что $\bar{\rho}\left(\pi_{0}\right)=\bar{\rho}_{0}$.

Пусть $\left\{\pi_{i}^{n}, \rho_{i}^{n}\right\}$ - аппроксимируюшая последовательность ансамблей такая, что соответствуюшая последовательность средних $\bar{\rho}^{n}$ сходится к состоянию $\bar{\rho}_{0}$. Представляя каждое состояние из ансамбля $\left\{\pi_{i}^{n}, \rho_{i}^{n}\right\}$ в виде счетной выпуклой комбинации чистых состояний, можно получить последовательность $\left\{\hat{\pi}_{j}^{n}, \hat{\rho}_{j}^{n}\right\}$ ансамблей из счетного числа чистых состояний с той же самой последовательностью средних состояний $\bar{\rho}^{n}$. Пусть $\widehat{\pi}^{n}$ - последовательность мер, приписывающих значение $\widehat{\pi}_{j}^{n}$ одноточечному множеству $\left\{\hat{\rho}_{j}^{n}\right\}$ при каждом $j$. Тогда

$$
\begin{aligned}
\chi_{\Phi}\left(\widehat{\pi}_{n}\right) & =\sum_{j} \widehat{\pi}_{j}^{n} H\left(\Phi\left(\hat{\rho}_{j}^{n}\right) \| \Phi\left(\bar{\rho}^{n}\right)\right) \geqslant \sum_{i} \pi_{i}^{n} H\left(\Phi\left(\rho_{i}^{n}\right) \| \Phi\left(\bar{\rho}^{n}\right)\right) \\
& =\chi_{\Phi}\left(\left\{\pi_{i}^{n}, \rho_{i}^{n}\right\}\right),
\end{aligned}
$$

где неравенство вытекает из выпуклости относительной энтропии. По построению $\operatorname{supp} \widehat{\pi}^{n} \subseteq \operatorname{Extr} \mathfrak{S}(\mathscr{H})$ для всех $n$. В силу предложения 2 
существует подпоследовательность $\widehat{\pi}^{n_{k}}$, сходящаяся к некоторой мере $\pi^{*}$ из $\mathscr{P} \mathscr{A}$. Поскольку множество $\operatorname{Extr} \mathfrak{S}(\mathscr{H})$ всех чистых состояний замкнуто в $\mathfrak{S}(\mathscr{H})^{4)}$, получаем, что $\operatorname{supp} \pi^{*} \subseteq \operatorname{Extr} \mathfrak{S}(\mathscr{H})$ в силу теоремы 6.1 из [13]. Ясно, что $\bar{\rho}\left(\pi^{*}\right)=\bar{\rho}_{0}$, и поэтому, как показано выше, сужение функционала $\chi_{\Phi}(\pi)$ на множество $\mathscr{P}_{\mathscr{A}}$ непрерывно в точке $\pi^{*}$. Эта непрерывность вместе с аппроксимирующим свойством последовательности $\left\{\pi_{i}^{n}, \rho_{i}^{n}\right\}$ и (12) дает

$$
\bar{C}(\Phi ; \mathscr{A})=\lim _{k \rightarrow \infty} \chi_{\Phi}\left(\left\{\pi_{i}^{n_{k}}, \rho_{i}^{n_{k}}\right\}\right) \leqslant \lim _{k \rightarrow \infty} \chi_{\Phi}\left(\widehat{\pi}_{n_{k}}\right)=\chi_{\Phi}\left(\pi^{*}\right) .
$$

Поскольку обратное неравенство следует из следствия 1 , получаем $\bar{C}(\Phi ; \mathscr{A})=\chi_{\Phi}\left(\pi^{*}\right)$, а значит, мера $\pi^{*}$ является оптимальным обобщенным ансамблем для $\mathscr{A}$-ограниченного канала $\Phi$. Теорема доказана.

Следствие 2. Для любого $\rho_{0}$ такого, что $H\left(\Phi\left(\rho_{0}\right)\right)<+\infty$, существует обобщенный ансамбль ${ }^{5)} \pi_{0}$ такой, что $\bar{\rho}\left(\pi_{0}\right)=\rho_{0} u$

$$
\chi_{\Phi}\left(\rho_{0}\right) \equiv \sup _{\sum_{i} \pi_{i} \rho_{i}=\rho_{0}} \chi_{\Phi}\left(\left\{\pi_{i}, \rho_{i}\right\}\right)=\int_{\mathfrak{S}(\mathscr{H})} H\left(\Phi(\rho) \| \Phi\left(\rho_{0}\right)\right) \pi_{0}(d \rho) .
$$

Д ок а затель с т о. Условие непрерывности в теореме очевидно выполняется для множества $\mathscr{A}=\left\{\rho_{0}\right\}$.

В конечномерном случае имеет место равенство

$$
\bar{C}(\Phi ; \mathscr{A})=\chi_{\Phi}(\bar{\rho})
$$

где $\bar{\rho}$ - среднее любого оптимального ансамбля. Обобщение этого равенства на бесконечномерный случай тесно связано с вопросом существования обобщенного оптимального ансамбля.

Следствие 3. Если для $\mathscr{A}$-ограниченного канала $\Phi$ существует обобщенньй оптимальньй ансамбль, то равенство (13) имеет место для некоторого оптимального среднего состояния $\bar{\rho}$.

Если для $\mathscr{A}$-ограниченного канала $\Phi$ равенство (13) выполнено для некоторого оптимального среднего $\bar{\rho}$ такого, что $H(\Phi(\bar{\rho}))<+\infty$, то существует обобщенный оптимальный ансамбль для $\mathscr{A}$-ограниченного канала $\Phi$.

Д о к а з а т ль с т о. Первое утверждение очевидно. Второе вытекает из следствия 2 .

З а м е ч а н и е. В приложении В показано, что условие непрерывности в теореме существенно. Можно показать, что это условие выполнено, если множество $\mathscr{A}$ выпукло и имеет конечное число крайних точек

4) Множество $\operatorname{Extr} \mathfrak{S}(\mathscr{H})$ определяется неравенством $H(\rho) \leqslant 0$ и поэтому замкнуто в силу полунепрерывности снизу квантовой энтропии.

5) Ниже будем считать, что обобщенный ансамбль - это мера с носителем на множестве чистых состояний. 
с конечной выходной энтропией. Существует предположение, что это условие выполнено и для произвольного выпуклого компактного множества $\mathscr{A}$ благодаря специальным свойствам оптимальных средних, рассмотренным в [17].

Предложение 3. Пусть $\mathscr{A}-$ компактное множество и $H^{\prime}$ есть $\mathfrak{H}$-оператор на пространстве $\mathscr{H}^{\prime}$ такой, что

$$
\operatorname{Tr} \exp \left(-\beta H^{\prime}\right)<+\infty \quad \text { для всех } \beta>0
$$

$u \operatorname{Tr} \Phi(\rho) H^{\prime} \leqslant h^{\prime} \partial л я$ всех $\rho \in \mathscr{A}$. Тогда для $\mathscr{A}$-ограниченного канала $\Phi$ существует обобщенный оптимальный ансамбль.

Д о к а з а т е л ь с т в. Покажем, что при выполнении условий данного предложения сужение выходной энтропии $H(\Phi(\rho))$ на множество $\mathscr{A}$ непрерывно, что гарантирует выполнение условия теоремы.

Пусть $\rho_{\beta}^{\prime}=\left(\operatorname{Tr} \exp \left(-\beta H^{\prime}\right)\right)^{-1} \exp \left(-\beta H^{\prime}\right)$ - состояние из $\mathfrak{S}\left(\mathscr{H}^{\prime}\right)$. Для любого $\rho$ из $\mathscr{A}$ имеем

$$
H\left(\Phi(\rho) \| \rho_{\beta}^{\prime}\right)=-H(\Phi(\rho))+\beta \operatorname{Tr} \Phi(\rho) H^{\prime}+\log \operatorname{Tr} \exp \left(-\beta H^{\prime}\right) .
$$

Пусть $\left\{\rho_{n}\right\}-$ произвольная последовательность состояний из $\mathscr{A}$, сходящаяся к состоянию $\rho$. Используя (15) и полунепрерывность снизу относительной энтропии, получаем

$$
\begin{gathered}
\limsup _{n \rightarrow \infty} H\left(\Phi\left(\rho_{n}\right)\right)=H(\Phi(\rho))+H\left(\Phi(\rho) \| \rho_{\beta}^{\prime}\right)-\liminf _{n \rightarrow \infty} H\left(\Phi\left(\rho_{n}\right) \| \rho_{\beta}^{\prime}\right) \\
+\underset{n \rightarrow \infty}{\limsup } \beta \operatorname{Tr} \Phi\left(\rho_{n}\right) H^{\prime}-\beta \operatorname{Tr} \Phi(\rho) H^{\prime} \leqslant H(\Phi(\rho))+\beta h^{\prime} .
\end{gathered}
$$

Устремляя $\beta$ в данном неравенстве к нулю, можно установить полунепрерывность сверху сужения функции $H(\Phi(\rho))$ на множество $\mathscr{A}$. Поскольку полунепрерывность снизу этой функции следует из полунепрерывности снизу квантовой энтропии (см. [19]), сужение функции $H(\Phi(\rho))$ на множество $\mathscr{A}$ непрерывно. Предложение 3 доказано.

Условия предложения 3 выполнены для гауссовских каналов с ограничением на среднюю энергию, имеющим вид (9), где $H=R^{T} \epsilon R$ гамильтониан системы осцилляторов с невырожденной матрицей энергии $\epsilon$, а $R$ - канонические переменные системы. Дадим набросок доказательства. Пусть

$$
R^{\prime}=K R+K_{E} R_{E}
$$

- уравнение канала в представлении Гейзенберга, в котором $R_{E}$ - канонические переменные «окружения», находящегося в гауссовском состоянии с нулевым средним и корреляционной матрицей $\alpha_{E}$ (см. [9]). Взяв $H^{\prime}=c\left[R^{T} R-I \operatorname{Sp} \alpha_{E} K_{E}^{T} K_{E}\right]$, где Sp обозначает след матрицы, получаем $\Phi^{*}\left(H^{\prime}\right)=c R^{T} K^{T} K R$ и всегда можно выбрать положительное $c$ так, что $\Phi^{*}\left(H^{\prime}\right) \leqslant H$. Более того, $H^{\prime}$ удовлетворяет условию (14). Таким образом, условия предложения 3 выполнены. 
Гипотеза. Для любого гауссовского канала с ограниченной средней энергией оптимальный обобщенный ансамбль представляет собой гауссовскую меру с носителем на множестве чистых гауссовских состояний с произвольным средним и фиксированной корреляиионной матричей.

Эта гипотеза была сформулирована в [9] для канала, описывающего усилитель/аттенюатор с классическим шумом. В случае чистого канала с затуханием, характерным свойством которого является нулевая минимальная выходная энтропия, справедливость этой гипотезы была установлена в [4]. Отметим еще, что классическим аналогом этого утверждения является гауссовость оптимального входного распределения вероятностей для гауссовского канала с квадратичным ограничением на входе.

5. Выпуклые ограничения. Квантовые каналы с ограничениями, задаваемыми выпуклыми множествами, обладают рядом специальных свойств, таких как единственность образа оптимального среднего (см. [17]). Следуюшая лемма дает континуальный аналог тождества Дональда [3].

Лемма 4. Для любой мерь $\pi$ из $\mathscr{P}$ и произвольного состояния $\sigma$ из $\mathfrak{S}(\mathscr{H})$ имеет место равенство

$\int_{\mathfrak{S}(\mathscr{H})} H(\rho \| \sigma) \pi(d \rho)=\int_{\mathfrak{S}(\mathscr{H})} H(\rho \| \bar{\rho}(\pi)) \pi(d \rho)+H(\bar{\rho}(\pi) \| \sigma)$.

Д о к а з а т е л ь с т в о. Заметим, что в конечномерном случае тождество Дональда

$$
\sum_{i} \pi_{i} H\left(\rho_{i} \| \sigma\right)=\sum_{i} \pi_{i} H\left(\rho_{i} \| \bar{\rho}(\pi)\right)+H(\bar{\rho}(\pi) \| \sigma)
$$

имеет место для положительных операторов с произвольным следом при использовании определения (2) относительной энтропии. Это тождество легко обобщается на случай обобщенных ансамблей в конечномерном гильбертовом пространстве, приводя к равенству (16). Таким образом, это равенство справедливо для операторов $P_{n} \rho P_{n}, P_{n} \sigma P_{n}$, где $P_{n}-$ произвольная последовательность проекторов конечного ранга, возрастающая к $I_{\mathscr{H}}$. Переходя к пределу $n \rightarrow \infty$ и используя теорему о монотонной сходимости, получаем (16) в бесконечномерном случае. Лемма 4 доказана.

Следуюшее предложение является обобшением «свойства максимальной удаленности», см. [10, предложение 1].

Предложение 4. Пусть $\mathscr{A}-$ выпуклое подмножество $\mathfrak{S}(\mathscr{H})$. Мера $\pi \in \mathscr{P}_{\mathscr{A}}$ является оптимальным обобщенным ансамблем для $\mathscr{A}$ - 
ограниченного канала $\Phi$ тогда и только, когда

$$
\int_{\mathfrak{S}(\mathscr{H})} H(\Phi(\rho) \| \Phi(\bar{\rho}(\pi))) \mu(d \rho) \leqslant \int_{\mathfrak{S}(\mathscr{H})} H(\Phi(\rho) \| \Phi(\bar{\rho}(\pi))) \pi(d \rho)=\chi_{\Phi}(\pi)
$$

для любой мерьи $\mu \in \mathscr{P}_{\mathscr{A}}$.

Д о к а з а т е л ь с в о. Пусть неравенство (17) выполнено для произвольной меры $\mu \in \mathscr{P}_{\mathscr{A}}$. В силу леммы 4

$$
\begin{aligned}
\chi_{\Phi}(\mu) & \leqslant \int_{\mathfrak{S}(\mathscr{H})} H(\Phi(\rho) \| \Phi(\bar{\rho}(\mu))) \mu(d \rho)+H(\Phi(\bar{\rho}(\mu)) \| \Phi(\bar{\rho}(\pi))) \\
& =\int_{\mathfrak{S}(\mathscr{H})} H(\Phi(\rho) \| \Phi(\bar{\rho}(\pi))) \mu(d \rho) \leqslant \chi_{\Phi}(\pi),
\end{aligned}
$$

что означает оптимальность меры $\pi$.

Обратно, пусть $\pi$ - оптимальный обобшенный ансамбль для $\mathscr{A}$ ограниченного канала $\Phi$ и $\mu-$ произвольная мера из $\mathscr{P} \mathscr{A}$. В силу выпуклости множества $\mathscr{A}$ мера $\pi_{\eta}=\eta \mu+(1-\eta) \pi$ также лежит в $\mathscr{P} \mathscr{A}$ при любом $\eta \in(0,1)$. Используя лемму 4 , получаем

$$
\begin{aligned}
\chi_{\Phi}(\pi) \geqslant \chi_{\Phi}\left(\pi_{\eta}\right)= & \int_{\mathfrak{S}(\mathscr{H})} H\left(\Phi(\rho) \| \Phi\left(\bar{\rho}\left(\pi_{\eta}\right)\right)\right) \pi_{\eta}(d \rho) \\
= & \eta \int_{\mathfrak{S}(\mathscr{H})} H\left(\Phi(\rho) \| \Phi\left(\bar{\rho}\left(\pi_{\eta}\right)\right)\right) \mu(d \rho) \\
& +(1-\eta) \chi_{\Phi}(\pi)+(1-\eta) H\left(\bar{\rho}(\pi) \| \bar{\rho}\left(\pi_{\eta}\right)\right) .
\end{aligned}
$$

В силу неотрицательности относительной энтропии имеем

$$
\int_{\mathfrak{S}(\mathscr{H})} H\left(\Phi(\rho) \| \Phi\left(\bar{\rho}\left(\pi_{\eta}\right)\right)\right) \mu(d \rho) \leqslant \chi_{\Phi}(\pi) .
$$

Используя полунепрерывность снизу относительной энтропии, получаем

$$
\begin{gathered}
\liminf _{\eta \rightarrow 0} \int_{\mathfrak{S}(\mathscr{H})} H\left(\Phi(\rho) \| \Phi\left(\bar{\rho}\left(\pi_{\eta}\right)\right)\right) \mu(d \rho) \\
\geqslant \int_{\mathfrak{S}(\mathscr{H})} \liminf _{\eta \rightarrow 0} H\left(\Phi(\rho) \| \Phi\left(\bar{\rho}\left(\pi_{\eta}\right)\right)\right) \mu(d \rho) \\
\geqslant \int_{\mathfrak{S}(\mathscr{H})} H(\Phi(\rho) \| \Phi(\bar{\rho}(\pi))) \mu(d \rho) .
\end{gathered}
$$

Предложение 4 доказано.

\section{6. Приложения.}

А. Теорема (см. [1]). Пусть $\rho_{n}-$ последовательность положительньх ядерных операторов, сходящаяся $\kappa$ оператору $\rho$ в слабой операторной топологии, такая, ито $\lim _{n \rightarrow \infty} \operatorname{Tr} \rho_{n}=\operatorname{Tr} \rho$. Тогда последовательность $\rho_{n}$ сходится $\kappa \rho$ по следовой норме.

Из этой теоремы следует, что если последовательность квантовых состояний сходится в слабой операторной топологии к состоянию, то 
эта последовательность сходится к данному состоянию и по следовой норме. Это утверждение можно рассматривать как некоммутативное обобщение того факта, что слабая сходимость распределений вероятностей на дискретном вероятностном пространстве к распределению вероятностей влечет $l_{1}$-сходимость. Используя эту теорему, можно обобщить критерий компактности из [14] (некоммутативный аналог теоремы Прохорова) на случай топологии, определяемой следовой нормой.

Критерий компактности. Замкнутое подмножество $\mathscr{K}$ множества $\mathfrak{S}(\mathscr{H})$ с топологией, определяемой следовой нормой, компактно тогда и только тогда, когда для любого $\varepsilon>0$ существует проектор конечного ранга $P_{\varepsilon}$ такой, что $\operatorname{Tr} P_{\varepsilon} \rho>1-\varepsilon \partial л я$ всех $\rho \in \mathscr{K}$.

Д ок а з а т ль с т о. Пусть $\mathscr{K}$ - компактное подмножество $\mathfrak{S}(\mathscr{H})$. Предположим, что существует такое $\varepsilon>0$, что для любого проектора конечного ранга $P$ найдется состояние $\rho \in \mathscr{K}$, у которого $\operatorname{Tr} P \rho \leqslant 1-\varepsilon$. Пусть $P_{n}$ - последовательность проекторов конечного ранга в $\mathscr{H}$, монотонно возрастающая к тождественному оператору $I_{\mathscr{H}}$ в слабой операторной топологии, а $\rho_{n}$ - соответствующая последовательность состояний из $\mathscr{K}$. В силу компактности существует подпоследовательность $\rho_{n_{k}}$, сходящаяся к состоянию $\rho_{*} \in \mathscr{K}$. По построению

$$
\operatorname{Tr} P_{n_{l}} \rho_{n_{k}} \leqslant \operatorname{Tr} P_{n_{k}} \rho_{n_{k}} \leqslant 1-\varepsilon \quad \text { для всех } k>l .
$$

Следовательно,

$$
\operatorname{Tr} \rho_{*}=\lim _{l \rightarrow+\infty} \operatorname{Tr} P_{n_{l}} \rho_{*}=\lim _{l \rightarrow+\infty} \lim _{k \rightarrow+\infty} \operatorname{Tr} P_{n_{l}} \rho_{n_{k}} \leqslant 1-\varepsilon,
$$

что противоречит $\rho_{*} \in \mathscr{K} \subseteq \mathfrak{S}(\mathscr{H})$.

Обратно, пусть $\mathscr{K}$ - замкнутое подмножество в $\mathfrak{S}(\mathscr{H})$, удовлетворяющее указанному критерию. Пусть $\rho_{n}$ - произвольная последовательность состояний из $\mathscr{K}$. Поскольку единичный шар в $\mathfrak{B}(\mathscr{H})$ компактен в слабой операторной топологии, существует подпоследовательность $\rho_{n_{k}}$, сходящаяся к положительному оператору $\rho_{*}$ в слабой операторной топологии. В силу соотношений

$$
\operatorname{Tr} \rho_{*} \leqslant \liminf _{k \rightarrow \infty} \operatorname{Tr} \rho_{n_{k}}=1
$$

для доказательства того, что оператор $\rho_{*}$ является состоянием, достаточно показать, что $\operatorname{Tr} \rho_{*} \geqslant 1$. Пусть $\varepsilon>0$ и $P_{\varepsilon}-$ соответствующий проектор. Тогда

$$
\operatorname{Tr} \rho_{*} \geqslant \operatorname{Tr} P_{\varepsilon} \rho_{*}=\lim _{k \rightarrow \infty} \operatorname{Tr} P_{\varepsilon} \rho_{n_{k}}>1-\varepsilon,
$$

где равенство следует из конечномерности пространства $P_{\varepsilon}(\mathscr{H})$. Таким образом, $\rho_{*}$ - состояние. Приведенная выше теорема гарантирует сходимость подпоследовательности $\rho_{n_{k}}$ к состоянию $\rho_{*}$ по следовой норме. Компакность подмножества $\mathscr{K}$ доказана. 
Б. Доказательство леммы 1. Заметим прежде всего, что если $\operatorname{supp} \pi \subseteq U$ для некоторого замкнутого выпуклого множества $U$, то

$$
\bar{\rho}(\pi) \in U .
$$

Это очевидно для любой меры $\pi$ с конечным носителем. По теореме 6.3 из [13] множество таких мер плотно в $\mathscr{P}$. Непрерывность отображения $\pi \mapsto \bar{\rho}(\pi)$ завершает доказательство (19).

Рассмотрим произвольную меру $\pi$ из $\mathscr{P}$. Поскольку пространство $\mathfrak{S}(\mathscr{H})$ сепарабельно, для любого $n \in \mathbf{N}$ сушествует последовательность $\left\{A_{i}^{n}\right\}$ борелевских множеств с диаметром, не превосходящим $1 / n$, и таких, что $\mathfrak{S}(\mathscr{H})=\bigcup_{i} A_{i}^{n}, A_{i}^{n} \cap A_{j}^{n}=\varnothing$ при $j \neq i$. Пусть $m=m(n)-$ такое число, что $\sum_{i=m+1}^{+\infty} \pi\left(A_{i}^{n}\right)<1 / n$. Рассмотрим конечный набор борелевских множеств $\left\{\widehat{A}_{i}^{n}\right\}_{i=1}^{m+1}$, где $\widehat{A}_{i}^{n}=A_{i}^{n}$ для всех $i=1, \ldots, m$ и $\widehat{A}_{m+1}^{n}=\bigcup_{i=m+1}^{+\infty} A_{i}^{n}$. Получаем

$$
\bar{\rho}(\pi)=\sum_{i=1}^{m+1} \int_{\widehat{A}_{i}^{n}} \rho \pi(d \rho)=\sum_{i=1}^{m+1} \pi_{i}^{n} \rho_{i}^{n},
$$

где $\pi_{i}^{n}=\operatorname{Tr} \int_{\widehat{A}_{i}^{n}} \rho \pi(d \rho)=\pi\left(\widehat{A}_{i}^{n}\right)$ и $\rho_{i}^{n}=\left(\pi\left(\widehat{A}_{i}^{n}\right)\right)^{-1} \int_{\widehat{A}_{i}^{n}} \rho \pi(d \rho)$ (без ограничения общности можно считать, что $\left.\pi_{i}^{n}>0\right)$. Пусть $\pi^{n}-$ вероятностная мера на $\mathfrak{S}(\mathscr{H})$, приписывающая значение $\pi_{i}^{n}$ одноточечному множеству $\left\{\rho_{i}^{n}\right\}$. Из равенства $(20)$ следует $\bar{\rho}\left(\pi^{n}\right)=\bar{\rho}(\pi)$. Поскольку мера $\pi^{n}$ имеет конечный носитель при каждом $n$, для доказательства утверждения леммы достаточно показать слабую сходимость последовательности мер $\pi^{n}$ к мере $\pi$ при стремлении $n$ к $+\infty$. В силу теоремы 6.1 из [13] для этого необходимо показать, что

$$
\lim _{n \rightarrow+\infty} \int_{\mathfrak{S}(\mathscr{H})} f(\rho) \pi^{n}(d \rho)=\int_{\mathfrak{S}(\mathscr{H})} f(\rho) \pi(d \rho)
$$

для произвольной ограниченной равномерно непрерывной функции $f(\rho)$ на $\mathfrak{S}(\mathscr{H})$. Пусть $M_{f}=\sup _{\rho \in \mathfrak{S}(\mathscr{H})}|f(\rho)|$. Для любого $\varepsilon>0$ найдется такое число $n_{\varepsilon}$, что $\varepsilon n_{\varepsilon}>2 M_{f}$ и

$$
\sup _{\rho \in U\left(n_{\varepsilon}\right)} f(\rho)-\inf _{\rho \in U\left(n_{\varepsilon}\right)} f(\rho)<\varepsilon
$$

для любого замкнутого шара $U\left(n_{\varepsilon}\right)$ с диаметром $1 / n_{\varepsilon}$. Пусть $n \geqslant n_{\varepsilon}$. По построению множество $\widehat{A}_{i}^{n}$ содержится в некотором шаре $U_{i}(n)$ при каждом $i=1, \ldots, m$. В силу (19) состояние $\rho_{i}^{n}$ лежит в том же шаре $U_{i}(n)$. Поэтому

$$
\begin{aligned}
& \left|\int_{\mathfrak{S}(\mathscr{H})} f(\rho) \pi^{n}(d \rho)-\int_{\mathfrak{S}(\mathscr{H})} f(\rho) \pi(d \rho)\right| \leqslant \sum_{i=1}^{m+1} \int_{\widehat{A}_{i}^{n}}\left|f(\rho)-f\left(\rho_{i}\right)\right| \pi(d \rho) \\
& \quad \leqslant \varepsilon \sum_{i=1}^{m} \pi\left(\widehat{A}_{i}^{n}\right)+2 M_{f} \pi\left(\widehat{A}_{m+1}^{n}\right)<2 \varepsilon \quad \text { для всех } n \geqslant n_{\varepsilon} .
\end{aligned}
$$


В. Пример канала с ограничением, не имеющего оптимального обобщенного ансамбля. Мы дадим пример классического канала, который стандартным образом может быть продолжен до квантового. Рассмотрим абелеву алгебру фон Неймана $l_{\infty}$ и ее предсопряженное пространство $l_{1}$, которые можно считать состоящими из диагональных операторов в сепарабельном гильбертовом пространстве $l_{2}$. Пусть $\Phi-$ тождественный канал в $l_{1}$. Рассмотрим последовательность состояний (распределений вероятностей)

$$
\rho_{n}=\{1-q_{n}, \underbrace{n^{-1} q_{n}, n^{-1} q_{n}, \ldots, n^{-1} q_{n}}_{n}, 0,0, \ldots\},
$$

где $q_{n}$ - последовательность чисел из $[0,1]$, которую мы определим ниже. Заметим, что $\chi_{\Phi}\left(\rho_{n}\right)=H\left(\rho_{n}\right)=h_{2}\left(q_{n}\right)+q_{n} \log n$, где $h_{2}(x)=$ $-x \log x-(1-x) \log (1-x)$. Ниже будет показано, что существует такая последовательность $q_{n}$, что $\lim _{n \rightarrow+\infty} q_{n}=0$, а соответствующая последовательность $\chi_{\Phi}\left(\rho_{n}\right)=H\left(\rho_{n}\right)$ монотонно возрастает к 1 . Пусть $q_{n}$ такая последовательность; определим множество $\mathscr{A}$ как замыкание последовательности $\rho_{n}$, которое состоит из состояний $\rho_{n}$ и чистого состояния $\rho_{*}=\lim _{n \rightarrow+\infty} \rho_{n}=\{1,0,0, \ldots\}$. По определению и в силу указанной выше монотонности $\bar{C}(\Phi ; \mathscr{A})=\lim _{n \rightarrow+\infty} \chi_{\Phi}\left(\rho_{n}\right)=1$, в то время как $\rho_{*}$ - единственное оптимальное среднее для $\mathscr{A}$-ограниченного канала $\Phi$ и $\chi_{\Phi}\left(\rho_{*}\right)=H\left(\rho_{*}\right)=0$. Таким образом, получаем $\bar{C}(\Phi ; \mathscr{A})>\chi_{\Phi}\left(\rho_{*}\right)$ и из следствия 3 заключаем, что у $\mathscr{A}$-ограниченного канала $\Phi$ не существует оптимального обобщенного ансамбля.

Построим последовательность $q_{n}$ с указанными выше свойствами. Рассмотрим строго возрастающую функцию $f(x)=x(1-\ln x)$ на $[0,1]$. Легко видеть, что $f^{\prime}(x)=-\ln x$ и $f([0,1])=[0,1]$. Пусть $f^{-1}$ - обратная функция и $g(x)=x f^{-1}((\ln 2) / x)$ для всех $x \geqslant 1$. Заметим, что функция $g(x)$ неявно задается уравнением

$$
g\left(1-\ln \left(\frac{g}{x}\right)\right)=\ln 2 .
$$

Учитывая это, легко показать, что функция $g(x)$ удовлетворяет следующему дифференциальному уравнению:

$$
\ln \left(\frac{g}{x}\right) g^{\prime}=\frac{g}{x}
$$

Поскольку $g(x) / x=f^{-1}((\ln 2) / x)$, имеем $g(x) / x \in[0,1]$. Используя это включение, $(21)$ и (22), получаем, что $g(x) \in[0,1], \lim _{x \rightarrow+\infty} g(x)=0$ и $g^{\prime}(x)<0$. Рассмотрим функцию $H(x)=h_{2}(g(x))+g(x) \ln x$. В силу указанных выше свойств функции $g(x),(21)$ и $(22)$ получаем

$$
\lim _{x \rightarrow+\infty} H(x)=(\ln 2)^{-1} \lim _{x \rightarrow+\infty} g(x) \ln x=1
$$


И

$$
\begin{aligned}
H^{\prime}(x) & =(\ln 2)^{-1}\left(g^{\prime}(x) \ln (1-g(x))-g^{\prime}(x) \ln g(x)+g^{\prime}(x) \ln x+\frac{g(x)}{x}\right) \\
& =(\ln 2)^{-1} g^{\prime}(x) \log (1-g(x))>0 \quad \forall x>1 .
\end{aligned}
$$

Следовательно, функция $H(x)$ строго возрастает на $[1,+\infty)$ и стремится к 1 на бесконечности. Полагая $q_{n}=g(n)$, получаем последовательность с указанными выше свойствами.

\section{СПИСОК ЛИТЕРАТУРЫ}

1. Dell'Antonio G.F. On the limits of sequences of normal states. - Comm. Pure Appl. Math., 1967, v. 20, p. 413-429.

2. Биллингсли П. Сходимость вероятностных мер. М.: Наука, 1977, 352 с.

3. Donald M. J. Further results on the relative entropy. - Math. Proc. Cambridge Philos. Soc., 1987, v. 101, № 2, p. 363-374.

4. Giovannetti V., Guha S., Lloyd S., Maccone L., Shapiro J. H., Yuen H. P. Classical capacity of the lossy bosonic channels: the exact solution. - Phys. Rev. Lett., 2004, v. 92, 027902; e-print quant-ph/0308012.

5. Hille E., Phillips R.S. Functional Analysis and Semi-groups. Providence, RI: Amer. Math. Soc., 1957, 808 p.

6. Холево А.С. Введение в квантовую теорию информации. М.: МЦНМО, 2002, $126 \mathrm{c}$.

7. Холево А.С. Статистическая структура квантовой теории. М.-Ижевск: Ин-т компьют. исслед., 2003, 191 с.

8. Холево А.С. Классические пропускные способности квантового канала с ограничением на входе. - Теория. вероятн. и ее примен., 2003, т. 48, в. 2, с. 359-374.

9. Holevo A.S., Werner R.F. Evaluating capacities of bosonic Gaussian channels. Phys. Rev. A, 2001, v. 63, 032312.

10. Holevo A.S., Shirokov M. E. On Shor's channel extension and constrained channels. Comm. Math. Phys., 2004, v. 249, № 2, p. 417-430.

11. Lindblad G. Expectations and entropy inequalities for finite quantum systems. Comm. Math. Phys., 1974, v. 39, № 2, p. 111-119.

12. Прохоров Ю. В. Сходимость случайных процессов и предельные теоремы теории вероятностей. - Теория вероятн. и ее примен., 1956, т. 1, в. 2, с. 177-238.

13. Parthasarathy K. R. Probability Measures on Metric Spaces. New York-London: Academic Press, 1967, 276 p.

14. Сарымсаков Т. А. Введение в квантовую теорию вероятностей. Ташкент: Фан, $1985,184 \mathrm{c}$.

15. Schumacher B., Westmoreland M. Optimal signal ensembles. - E-print quant$\mathrm{ph} / 9912122$.

16. Serafini A., Eisert J., Wolf M.M. Multiplicativity of maximal output purities of Gaussian channels under Gaussian inputs. - E-print quant-ph/0406065, 2004.

17. Shirokov M. E. The Holevo capacity of infinite dimensional channels. - E-print quantph/0408009, 2004.

18. Shor $P$. $W$. Equivalence of additivity questions in quantum information theory. Comm. Math. Phys., 2004, v. 246, № 3, p. 453-472.

19. Wehrl A. General properties of entropy. - Rev. Modern Phys., 1978, v. 50, № 2, p. 221-260. 